Check for updates

Cite this: Mater. Adv., 2021, 2, 3014

Received 27th February 2021, Accepted 19th March 2021

DOI: 10.1039/d1ma00170a

rsc.li/materials-advances

\title{
Fabrication of chiral polydiacetylene nanotubes via supramolecular gelation of a triterpenoid- derived amphiphile $\dagger$
}

\begin{abstract}
Xia Yu, (D) ${ }^{a}$ Hao Zhang, ${ }^{a}$ Yuxia Gao, ${ }^{b}$ Jun Hu (D)*a and Min-Hui Li (D)*ac
Polydiacetylenes (PDA) can undergo a blue-to-red color transition in response to external stimuli because of the extensive delocalized $\pi$-electron networks and intrinsic conformational restrictions, which make PDA particularly attractive building blocks for stimuli-responsive materials. In this work, a blue chiral C4-MOP/PDA gel was fabricated successfully by UV irradiating a white supramolecular gel that was assembled from a triterpenoid-derived gelator (C4-MOP) and a diacetylene monomer (PCDA). This photopolymerization was completed within $5 \mathrm{~min}$ with the generation of the new negative Cotton effect at $657 \mathrm{~nm}$ in the visible blue light region, which strongly revealed the transmission, amplification and visualization of molecular chirality during this gelation and the photopolymerization process. Notably, the nanotubular microstructures of the white gel C4-MOP/PCDA that curled from twisted ribbons barely changed after polymerization into C4-MOP/PDA. In addition, because C4-MOP/PDA contained an alternating ene-acetylene conjugated structure, it exhibited multiple color transitions to external stimuli including temperature, organic solvent, and mechanical force. This work provides chiral polydiacetylene assemblies by using natural triterpenoid gelators, and may open new developments in the design of smart responsive chiral PDA materials.
\end{abstract}

\section{Introduction}

Polydiacetylenes (PDA) are a type of highly conjugated polymer and normally formed via the topochemical 1,4-polymerization of diacetylene (DA) monomers upon UV light or $\gamma$-ray irradiation. ${ }^{1-3}$ Because of the extensive delocalized $\pi$-electron networks and intrinsic conformational restrictions, PDA can undergo a blue-to-red color transition in response to external stimuli like temperature, ${ }^{4}$ organic solvents, ${ }^{5} \mathrm{pH},{ }^{6}$ and mechanical forces, ${ }^{7}$ thus showing intriguing colorimetric and optical properties. These distinctive advantages make PDA particularly attractive building blocks for stimuli-responsive functional materials. ${ }^{8-11}$ It should be mentioned that an appropriate

\footnotetext{
${ }^{a}$ Beijing Advanced Innovation Center for Soft Matter Science and Engineering, Beijing University of Chemical Technology, Beijing 100029, China. E-mail: jhu@mail.buct.edu.cn

${ }^{b}$ Department of Applied Chemistry, College of Science, China Agricultural University, Beijing 100193, China

${ }^{c}$ Chimie ParisTech, PSL University Paris, CNRS, Institut de Recherche de Chimie Paris, Paris 75005, France.E-mail: min-hui.li@chimieparistech.psl.eu

$\dagger$ Electronic supplementary information (ESI) available: Detailed statistical results of assembled nanostructures; TEM images of nanotubes; intermolecular forces of C4-MOP single crystal structure; $d$-spacing from XRD; UV-vis spectra and fluorescence spectra of C4-MOP/PDA before and after external stimuli. CCDC 2057123. For ESI and crystallographic data in CIF or other electronic format see DOI: $10.1039 / \mathrm{d} 1 \mathrm{ma} 00170 \mathrm{a}$
}

orderly arrangement of DA monomers is often required for the topochemical polymerization of PDA. ${ }^{12,13}$ So far, two common strategies have been reported for promoting the regular packing of DA monomers. One is to covalently attach functional groups to DA backbone for the synthesis of amphiphilic DA-tailored molecules. For example, Sureshan and co-workers have developed an organic gelator by conjugating 4,6-Obenzylidene $\beta$-D-galactopyranoside to the DA skeleton, ${ }^{14}$ and found that this gelator can adhere to fabrics to generate semiconducting PDA materials upon UV-irradiation. By contrast, another strategy is to noncovalently co-assemble DA monomers with small molecules. For example, Liu et al. have co-assembled a chiral L-histidine ester derivative with the DA monomer into a spiral nano-broadband supramolecular gel, which was easily polymerized into PDA upon UV-irradiation. ${ }^{15}$ This PDA not only had an excellent colorimetric response, but also exhibited a strong circular dichroism signal and circularly polarized luminescence. Obviously, compared with the former strategy involving covalent chemical modifications, the latter one is simple and convenient because the noncovalent physical packing can avoid time-consuming synthesis tasks and expand the selection range of small molecules. ${ }^{16,17}$ It should be pointed out here that the existence of a suitable co-assembly partner with the DA monomer is the key point in the latter strategy. However, as far as we know, chiral partners remain limited. Thus, searching for chiral molecules with a 


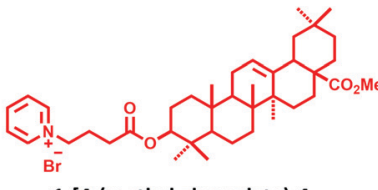

1-[4-(methyl oleanolate)-4-

oxobutyl]pyridinium bromide, C4-MOP

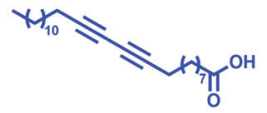

10,12-pentacosadiynoic acid, PCDA

Scheme 1 Molecular structures of co-gelator C4-MOP and PCDA.

suitable assembly ability will enrich the library of co-assembly partners with DA monomers and benefit the production of diverse PDA.

Triterpenoids, consisting of six isoprene units, are abundant in many plants in the form of free acids or aglycones. They possess unique rigid chiral skeletons, multiple reactive sites, and good biocompatibility. ${ }^{18,19}$ These features allow for the easy modification and stacking of rigid skeletons, thus rendering triterpenoids great supramolecular gelators. ${ }^{20,21}$ For example, Bag's group have found that triterpenoids like corosolic acid, betulin, and ursolic acid can assemble into supramolecular gels with different spherical, flower-like, fibrous, and vesicular microstructures; ${ }^{22-24}$ Cienfuegos et al. modified oleanolic acid with the benzotriazole moiety and prepared a homogeneous stiff gel when capturing atmospheric water. ${ }^{25}$ Clearly, their intrinsic good assembly ability and multiple reactive sites make triterpenoids ideal candidates as chiral co-assembly partners with DA monomers.

Herein, in this research a triterpenoid-derived gelator (C4-MOP, Scheme 1) consisting of hydrophobic methyl oleanonate and a hydrophilic pyridinium terminal group was synthesized to coassemble with the diacetylene monomer (10,12-pentacosadiynoic acid, PCDA, Scheme 1) for the fabrication of orderly assemblies, followed by topochemical photopolymerization, since C4-MOP can be regulated into twisted ribbons in methanol/water mixed solvents. ${ }^{26}$ The results showed that C4-MOP/PCDA formed a stable white gel in methanol/water mixed solvents, and upon UV irradiation this gel underwent a noticeable white-to-blue color change with the generation of C4-MOP/PDA via the 1,4-photopolymerization of PCDA. During this gelation and polymerization process, the transmission and visualization of molecular chirality were achieved successfully with the appearance of a negative Cotton effect at $657 \mathrm{~nm}$ in the visible blue light region. The assembled nanotubular microstructures of the white gel that curled from helical ribbons barely changed after polymerization. It is known that many supramolecular templates have been used for coassembly with DA monomers, followed by post-polymerization, for the immobilization of these ordered superstructures ${ }^{27}$ whereas only a few of them, like polysaccharides, amino acids and triazoles, have achieved in situ chirality transcription and amplification. ${ }^{15,28-30}$ This C4-MOP/PCDA-dictated transmission and visualization of molecular chirality is a vital supplement for the post-polymerization of preorganized chiral assemblies. In addition, because C4-MOP/PDA contains an alternating ene-acetylene conjugated structure, it exhibited color transitions to external stimuli including temperature, organic solvent, and mechanical force. Our chiral PDA assemblies dictated by the natural triterpenoid gelator might be expected to open new developments in the design of smart PDA materials.

\section{Experimental}

\section{Materials}

10,12-Pentacosadiynoic acid (PCDA), oleanolic acid (OA), methyl iodide $\left(\mathrm{CH}_{3} \mathrm{I}\right)$, 4-bromobutyryl chloride, dichloromethane (DCM), $\mathrm{N}, \mathrm{N}$-dimethylformamide (DMF), potassium carbonate $\left(\mathrm{K}_{2} \mathrm{CO}_{3}\right)$, pyridine and other reagents were local commercial products and used as received. Milli-Q water was used in all cases.

\section{Characterization}

${ }^{1} \mathrm{H}$ and ${ }^{13} \mathrm{C}$ nuclear magnetic resonance (NMR) spectra were recorded using a Bruker AVANCE III 400 spectrometer. UV-vis spectra were recorded in quartz cuvettes (light path $1 \mathrm{~mm}$ ) with a Hitachi U-3900 spectrophotometer. CD spectra were obtained using a Pistar $\pi-180$ instrument (Applied Photophysics Ltd, Leatherhead, UK) with a $150 \mathrm{~W}$ xenon lamp as the light source. Experiments were performed at room temperature in a quartz cell with a $1 \mathrm{~mm}$ path length over the range of 220-800 $\mathrm{nm}$. Transmission electron microscopy (TEM) was performed using a HT7700 instrument (Hitachi, Japan) with an $80 \mathrm{kV}$ acceleration voltage. Samples were prepared by drop-casting the sol onto a carbon-coated copper grid, and allowing this stand for about $1 \mathrm{~min}$. After that, the excess sample solution was wiped away using filter paper and dried in air. X-ray diffraction (XRD) measurements were recorded using an Ultima IV X-ray diffractometer (Rigaku Co., Tokyo, Japan) with $\mathrm{CuK} \alpha$ radiation ( $\lambda=1.5406 \AA)$, operating at a voltage of $40 \mathrm{kV}$ and a current of $40 \mathrm{~mA}$. X-ray diffraction data of single crystals was collected using an XtaLAB PRO 007HF (Mo) single crystal X-ray diffractometer (Rigaku, Japan). The extended packing plots and data from crystal packing were implemented using the Mercury 1.4.3 program. FT-IR spectra were recorded using a Nicolet 6700 FT-IR spectrometer (Thermo Fisher, USA) in situ. The test samples were prepared by mixing with potassium bromide and tabletting.

\section{Synthesis of C4-MOP}

C4-MOP was synthesized with a yield of $61 \%$ according to the previous literature. ${ }^{18}{ }^{1} \mathrm{H} \mathrm{NMR}$ (400 MHz, $\mathrm{CDCl}_{3}, \mathrm{ppm}$ ): $\delta=9.59-9.58$ (d, $2 \times 1 \mathrm{H}, J=4 \mathrm{~Hz}$, pyridinium- $\mathrm{H}), 8.50-8.46\left(\mathrm{t}, 1 \mathrm{H}, J_{1}=8 \mathrm{~Hz}\right.$, $J_{2}=8 \mathrm{~Hz}$, pyridinium- $\left.\mathrm{H}\right), 8.10-8.06\left(\mathrm{t}, 1 \mathrm{H}, J_{1}=8 \mathrm{~Hz}, J_{2}=8 \mathrm{~Hz}\right.$, pyridinium- $\mathrm{H}), 5.28-5.27(\mathrm{t}, 1 \mathrm{H}, \mathrm{J}=4 \mathrm{~Hz}, 12-\mathrm{CH}=\mathrm{C}), 5.20-5.16$ $\left(\mathrm{t}, 2 \mathrm{H}, J_{1}=8 \mathrm{~Hz}, J_{2}=8 \mathrm{~Hz}\right.$, pyridinium- $\left.\mathrm{CH}_{2}\right), 4.48-4.43(\mathrm{dd}, 1 \mathrm{H}$, $\left.J_{1}=8 \mathrm{~Hz}, J_{2}=8 \mathrm{~Hz},-\mathrm{COOCH}_{2}\right), 3.62\left(\mathrm{~s}, 3 \mathrm{H},-\mathrm{COOCH}_{3}\right), 2.60-2.57$ $\left(\mathrm{t}, 2 \mathrm{H}, J_{1}=8 \mathrm{~Hz}, J_{2}=4 \mathrm{~Hz},-\mathrm{CH}_{2} \mathrm{COO}\right), 1.63,1.12,0.92,0.89,0.84,0.72$ $\left(\mathrm{s}, 7 \times 3 \mathrm{H}, 7 \times \mathrm{CH}_{3}\right) \cdot{ }^{13} \mathrm{C} \mathrm{NMR}\left(100 \mathrm{MHz}, \mathrm{CDCl}_{3}, \mathrm{ppm}\right): \delta=178.4$, 172.5, 148.3, 145.5, 145.2, 144.0, 128.4, 122.3, 82.2, 60.9, 55.5, 51.7, 47.7, 46.9, 46.0, 41.8, 41.4, 39.4, 38.3, 37.9, 37.1, 34.0, 33.3, 32.7, 32.5, $30.8,30.5$, 28.4, 27.8, 27.4, 26.1, 23.8, 23.6, 23.2, 18.4, 17.0, 16.9, 15.5 .

\section{Cultivation of C4-MOP single crystal}

Initially, an appropriate amount of C4-MOP was dissolved in methylene chloride, and then methanol was added under the premise of keeping the solution clear. After that, it was allowed to stand at room temperature for the slow evaporation of the solvent. Consequently, the needle-like single crystals were 
obtained. Detailed data on the single crystals can be found in the ESI. $\dagger$

\section{Results and discussion}

Fabrication and photopolymerization of orderly structures via supramolecular gelation

As a general procedure, C4-MOP and PCDA (equivalent ratio 1:1) were initially dissolved in methanol to form a clear solution, followed by the gradual addition of water (methanol/ water $=1 / 2, \mathrm{v} / \mathrm{v}$ ). After heating at $90{ }^{\circ} \mathrm{C}$ for $5 \mathrm{~min}$, the solution was cooled to room temperature, and consequently a white supramolecular gel of C4-MOP/PCDA was formed (Fig. 1A), mainly driven by the synergistic effect of hydrophobic interactions, van der Waals forces, and $\mathrm{C}-\mathrm{H} \cdots \mathrm{O}$ interactions. The photopolymerization of C4-MOP/PCDA was investigated by means of UV light irradiation. As shown in Fig. 1A, upon exposure to UV light $(254 \mathrm{~nm}$, $10 \mathrm{~W}$ ), the white C4-MOP/PCDA gel rapidly turned to blue as a result of 1,4-polymerization. This indicated that the supramolecular gelation did help the photopolymerization of the diacetylene monomers. UV-vis absorption was employed to explore the kinetics process and structural changes. A new absorption band appeared at around $657 \mathrm{~nm}$ accompanied by a shoulder at $594 \mathrm{~nm}$ upon UV-irradiation (Fig. 1B), which clearly established the beginning of the structure transition from the co-assembled C4-MOP/PCDA to the conjugated alternating ene-yne C4-MOP/ PDA, which corresponded well with the generation of blue color in Fig. 1A. With the extension of the irradiation time, the intensity at $657 \mathrm{~nm}$ gradually increased until no significant change was observed after 5 min (Fig. 1C), which indicated complete photopolymerization.
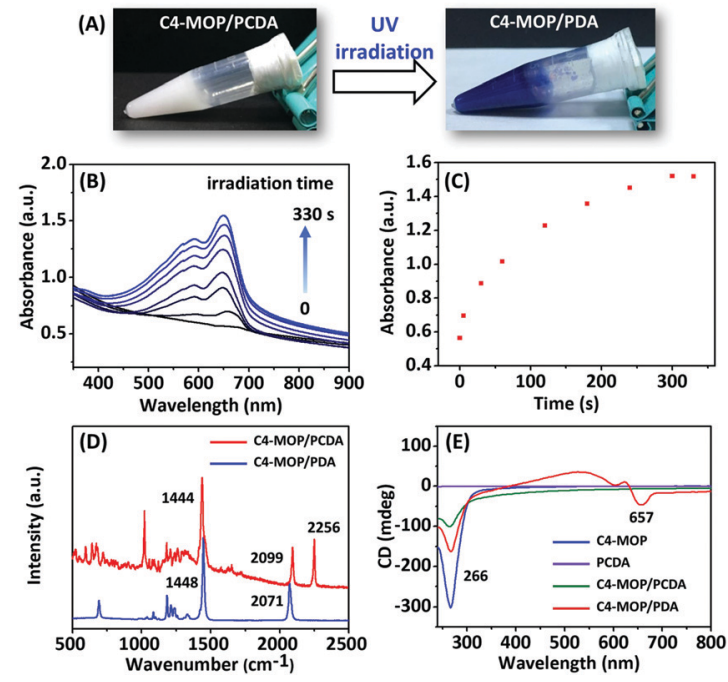

Fig. 1 (A) Digital photos of the white gel C4-MOP/PCDA and the blue gel C4-MOP/PDA after UV-irradiation (254 nm, $10 \mathrm{~W}$ ). (B) UV-vis spectra of C4-MOP/PCDA under UV-irradiation (254 nm, $10 \mathrm{~W}$ ) for different time. (C) Absorbance intensity of C4-MOP/PCDA at $657 \mathrm{~nm}$ as a function of the irradiation time. (D) Raman spectra of C4-MOP/PCDA and C4-MOP/PDA. (E) CD spectra of C4-MOP, PCDA, C4-MOP/PCDA and C4-MOP/PDA in methanol/water $\left(1 / 2, \mathrm{v}: \mathrm{v}, 5 \mathrm{mg} \mathrm{mL}^{-1}\right)$.
Further evidence for the formation of C4-MOP/PDA came from Raman spectroscopy. As shown in Fig. 1D, two bands associated with the conjugated ene-yne of C4-MOP/PDA at $2071 \mathrm{~cm}^{-1}(\mathrm{C} \equiv \mathrm{C})$ and $1448 \mathrm{~cm}^{-1}(\mathrm{C}=\mathrm{C})$ appeared, whereas the $\mathrm{C} \equiv \mathrm{C}$ band at $2256 \mathrm{~cm}^{-1}$ of $\mathbf{C 4}$-MOP/PCDA disappeared completely, which served as solid proof of topochemical 1, 4-polymerization. It should be pointed out that the generation of bands at 1444 and $2099 \mathrm{~cm}^{-1}$ for C4-MOP/PCDA was due to laser irradiation as the Raman spectrum was obtained. ${ }^{31}$ All of the above results indicated that the co-assembled C4-MOP/ PCDA gel afforded orderly assemblies, which in turn promoted the following photopolymerization process.

It is known that the co-assembly of achiral molecules with chiral molecules is an effective method for realizing chirality transfer and amplification. ${ }^{15,32}$ To evaluate whether the C4-MOP/PDA gel possessed optical activity, circular dichroism (CD) was carried out because the Cotton effect can be used as a sensitive chiral indicator. ${ }^{33}$ As shown in Fig. 1E, the chiral C4-MOP exhibited a negative Cotton effect at $266 \mathrm{~nm}$, whereas it was silent for achiral PCDA. Accordingly, no CD signal of the co-assembled C4-MOP/ PCDA was observed in the visible light region in addition to the negative Cotton effect at $266 \mathrm{~nm}$. Conversely, after UV light irradiation an additional negative Cotton effect at $657 \mathrm{~nm}$ was observed. The appearance of the CD signals in the visible blue light region was attributed to the ordered assembly of structures in white gel which transferred and amplified the chirality from chiral C4-MOP to C4-MOP/PDA. ${ }^{34}$ Obviously, C4-MOP offered a suitable chiral template to induce supramolecular chirality, which can be imprinted into the polymerized C4-MOP/PDA gel.

\section{Morphologies}

Transmission electron microscopy (TEM) was employed to investigate the morphologies of C4-MOP/PCDA before and after UV-irradiation. PCDA assembled into microsheets (Fig. 2A), whereas C4-MOP formed twisted ribbons with a width of around $0.3 \mu \mathrm{m}$ (Fig. 2B and Fig. S1A, ESI $\dagger$ ). Conversely, chiral nanotubes were observed for the white gel of C4-MOP/PCDA with a diameter of $0.8 \mu \mathrm{m}$, which was clearly curled from twisted ribbons (white arrows, Fig. 2C and Fig. S1B and S2, ESI $\dagger$ ). These fairly uniform nanotubes indicated that PCDA and C4-MOP co-assembled synergistically rather than forming nanostructures separately. During this process, the chirality was transferred and expressed on account of the rich chiral centers of C4-MOP. Notably, no significant change in morphology was observed after photopolymerization (white arrows, Fig. 2D and Fig. S1C and S2, ESI $\dagger$ ), probably resulting from the fact that the process was a topochemical reaction. ${ }^{15}$

\section{Possible packing patterns}

In order to reveal the packing structures, the single crystal analysis and power X-ray diffraction (XRD) experiments were carried out. We first used Chem 3D software to measure the molecular chain length of the fully extended PCDA molecule, which was $31.1 \AA$, as shown in Fig. S3A (ESI $\dagger$ ). Meanwhile, a single crystal of C4-MOP was successfully cultured in the mixed solvent of methanol/dichloromethane. The crystal structure 

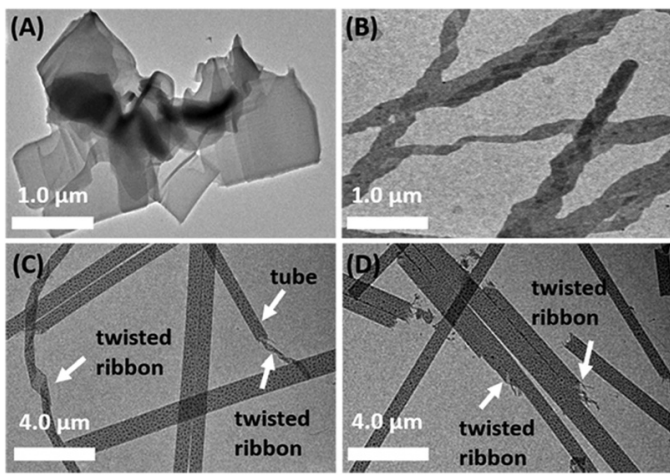

Fig. 2 TEM images of (A) PCDA, (B) C4-MOP, (C) C4-MOP/PCDA, and (D) C4-MOP/PDA in methanol/water (1/2, v:v, $\left.5 \mathrm{mg} \mathrm{mL}^{-1}\right)$.

and packing patterns are illustrated in Fig. 3, Fig. S4-S6 and Table S1 (ESI $\dagger$ ). The length of the C4-MOP molecule was $20.8 \AA$, and the packing diagram of C4-MOP revealed an orthorhombic structure, where two adjacent molecules with a head-to-tail stacking were regarded as repeating units (41.65 ̊) along the orientation c (Fig. 3). Moreover, two molecules were staggered and stacked in antiparallel fashion along the direction $\mathbf{b}$, and the distance between the two identical conformation molecules was $12.56 \AA$. As for the interactions between molecules, as depicted in Fig. S6 (ESI $\dagger$ ), the $\mathrm{C}-\mathrm{H} \cdots \mathrm{O}$ interaction (with a distance of $2.55 \AA$ ) and four types of $\mathrm{C}-\mathrm{H} \cdots \mathrm{Br}$ interactions (with distances of $2.69,2.87,2.73$, and $2.87 \AA$ ) were observed. All these intense multiple interactions helped to solidify the molecular conformations and stabilize the crystal structure.

The XRD results of C4-MOP, PCDA, and C4-MOP/PCDA assemblies are shown in Fig. 4 and Table S2 (ESI $\dagger$ ). The peaks of PCDA at 1.86, 5.55, 7.42, 9.28, 13.03, 16.83, and $22.87^{\circ}$ corresponding to $47.44,15.90,11.59,9.52,6.79,5.26$ and $3.91 \AA$ are observed in Fig. $4 \mathrm{~A}$, following the ratio of $1: 1 / 3: 1 / 4$ : $1 / 5: 1 / 7: 1 / 9: 1 / 12$. On the basis of this $d$-spacing (47.44 $\AA$ ) and the fully stretched molecular length of PCDA $\left(L_{0}=31.1 \AA\right.$, Fig. S3A, ESI $\dagger$ ), the tilting angle $(\theta)$ of PCDA molecules with respect to the lamellar plane was estimated to be $49^{\circ}$ in Fig. S3B (ESI $\dagger$ ). ${ }^{35,36}$ As for the C4-MOP assemblies, the reflection peaks appeared at $1.99,4.56,14.01,21.52$, and $23.88^{\circ}$ in Fig. 4B with
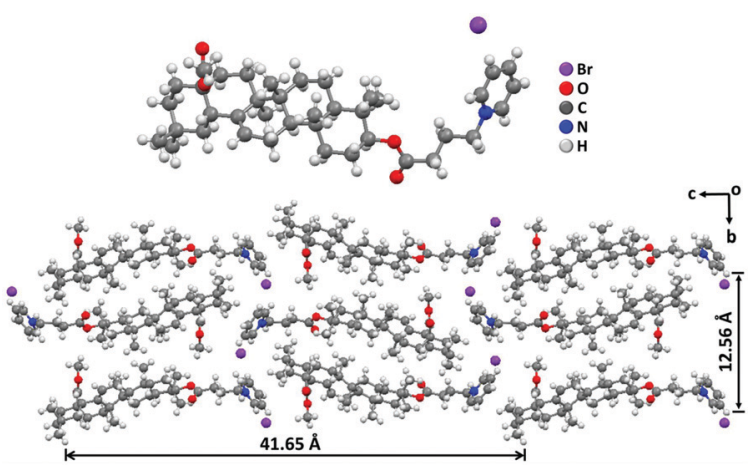

Fig. 3 X-ray single crystal structure of C4-MOP and its spatial arrangements along the directions $\mathbf{b}$ and $\mathbf{c}$. Purple: bromine atom, red: oxygen atom, gray: carbon atom, blue: nitrogen atom, white: hydrogen atom.
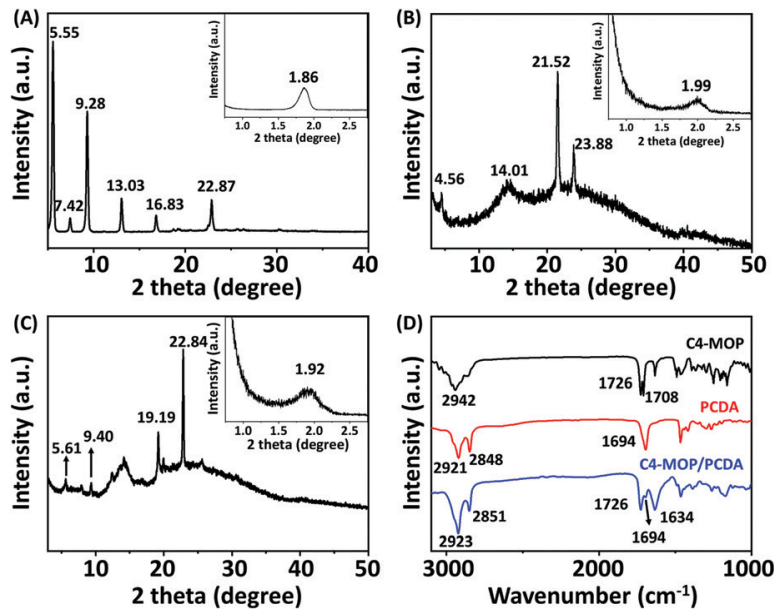

Fig. 4 XRD spectra of (A) PCDA, (B) C4-MOP, and (C) C4-MOP/PCDA assemblies. Insets in $(A, B$, and $C$ ) correspond to the results in the small angle region. (D) FT-IR spectra of PCDA, C4-MOP, and C4-MOP/PCDA assemblies.

$d$-spacings of $44.34,20.06,6.31,4.12$, and $3.72 \AA$, calculating the ratio of $1: 1 / 5: 1 / 7: 1 / 11: 1 / 13$. That is to say, the interlayer distance of C4-MOP was $44.34 \AA$. This value was almost identical to that of the repeating units in direction $\mathbf{c}$ of the C4-MOP crystal (41.65 $\AA$ ), suggesting that the packing mode adopted in the gel was similar to that in direction $\mathbf{c}$ of the single crystal. In addition, the reflection peaks of C4-MOP/PCDA were $1.92,5.61,9.40,19.19$, and $22.84^{\circ}$ with $d$-spacing of 45.96 , $15.73,9.40,4.62$, and $3.89 \AA$, following the ratio of $1: 1 / 3$ : $1 / 5: 1 / 10: 1 / 12$ (Fig. 4C). This strongly indicated that the lamellar structures were formed in the gel with a $d$ value of $45.96 \AA$. As this $d$ value is very close to that of the PCDA bilayer structure in Fig. 4A, it was assumed that C4-MOP was inserted into the bilayer structure of PCDA to form a structural unit with partial crossover between the hydrophobic part of C4-MOP and the flexible chain of PCDA.

Furthermore, the FT-IR spectra of PCDA, C4-MOP, and the xerogel of C4-MOP/PCDA were investigated (Fig. 4D). In the case of PCDA, the stretching vibration of $\mathrm{C}=\mathrm{O}$ corresponding to $\mathrm{COOH}$ appeared at $1694 \mathrm{~cm}^{-1}$, indicating that PCDA had a hydrogen-bonded carbonyl stretching band. ${ }^{37}$ The peaks at 2921 and $2848 \mathrm{~cm}^{-1}$ were the asymmetric and symmetric stretching vibrations of $\mathrm{CH}_{2}$, respectively. For C4-MOP, the peak at $2942 \mathrm{~cm}^{-1}$ was the asymmetric stretching vibration of $\mathrm{CH}_{2}$ and $\mathrm{CH}_{3}$, and the stretching vibrations at 1726 and $1708 \mathrm{~cm}^{-1}$ were assigned to $\mathrm{C}=\mathrm{O}$ groups in the ester groups at positions 3 and 17, respectively. In the co-assembled C4-MOP/ PCDA system, the stretching vibrations of $\mathrm{C}=\mathrm{O}$ shifted from 1708 to $1634 \mathrm{~cm}^{-1}$ upon gelation, whereas the original peaks at 1726 and $1694 \mathrm{~cm}^{-1}$ remained unchanged. This shift might originated from the hydrogen bonding between the $\mathrm{C}=\mathrm{O}$ in the ester group of C4-MOP and the carboxyl head group of PCDA. In a polar methanol/water environment, both the hydrophilic heads of C4-MOP and PCDA faced outward, while their hydrophobic backbones overlapped partially because of hydrophobic interactions. As a result, they preferentially arrange in a more 

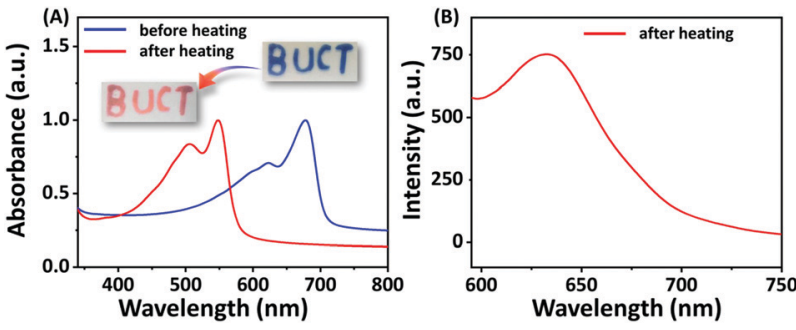

Fig. 5 (A) Normalized UV-vis spectra of C4-MOP/PDA film before and after heating. (B) Fluorescence spectra of C4-MOP/PDA film after heating. The insert "BUCT" pattern in (A) was prepared as follows: initially, a C4-MOP/PCDA film was obtained by drying C4-MOP/PCDA gel on a glass plate. After that, the film was covered by a mask with a "BUCT" pattern and then irradiated by UV light, thus affording a blue "BUCT" of C4-MOP/PDA The blue color immediately turn red when placed on a hot plate.

orderly manner to form intermolecular hydrogen bonding interactions. Also, asymmetric and symmetric stretching vibrations of $\mathrm{CH}_{2}$ appearing at 2923 and $2851 \mathrm{~cm}^{-1}$ suggested an ordered packing of alkyl chains primarily promoted by van der Waals forces. ${ }^{38,39}$ Apparently, the hydrophobic interactions, the intermolecular hydrogen bonding interactions, and van der Waals forces played crucial roles in the formation of the supramolecular gel C4-MOP/PCDA.

Based on the above results, a possible assembly process was proposed as follows: initially a few assembled dimers of C4-MOP and PCDA elongated to form twisted ribbons. Subsequently, in order to expose less hydrophobic areas in the hydrophilic environment, the twisted ribbons continuously curled and finally constructed the nanotubes. During the co-assembly process, the molecular chirality of C4-MOP was magnified and transmitted to C4-MOP/PCDA, as confirmed by the CD spectra in Fig. 1D and the TEM images in Fig. 2.

\section{Color transition}

Since C4-MOP/PDA contains an alternating ene-acetylene conjugated skeleton, we explored its color transitions with external stimuli including temperature, organic solvent, and mechanical force. As shown in Fig. 5A, the C4-MOP/PDA underwent a clear blue-to-red color transition when the film was heated, with an absorption shift from 678 and $623 \mathrm{~nm}$ to 548 and $506 \mathrm{~nm}$, respectively, in UV-vis spectra. Moreover, a fluorescence signal at $634 \mathrm{~nm}$ was generated as the red phase appeared (Fig. 5B). Similar to the phenomenon of thermochromism, both organic solvent and mechanical force could also induce the color change from blue to red. As shown in Fig. S7 (ESI $\dagger$ ), after external grinding or fuming by chloroform, the blue color of C4-MOP/PDA disappeared with the appearance of the red color, as well as the production of strong fluorescence. This phenomenon was due to the low conjugation length of PDA, which originated from the increased motional freedom of the PDA side chains when under the stimulus of temperature, organic solvent, or mechanical force. ${ }^{40,41}$ In brief, this color transition of C4-MOP/PDA observed by the naked eye could be utilized for the preparation of multifunctional sensors for external stimuli.

\section{Conclusions}

In summary, a stable white supramolecular gel C4-MOP/PCDA was formed in methanol/water mixed solvents by coassembling PCDA with C4-MOP, which can be photopolymerized into C4-MOP/PDA upon UV-irradiation, accompanied by a color change of the gel from white to blue. UV-vis and Raman spectra revealed that the photopolymerization was completed within $5 \mathrm{~min}$, and a possible packing pattern was proposed by measuring the X-ray diffraction and FT-IR spectra. In addition, the new negative Cotton effect at $657 \mathrm{~nm}$ in the visible blue light region in the CD spectra indicated that the transmission and visualization of molecular chirality have been achieved successfully during the gelation and polymerization process. Notably, the assembled nanotubes of C4-MOP/PCDA that curled from twisted ribbons barely changed after polymerization into C4-MOP/PDA. Furthermore, because of the alternating ene-acetylene conjugated structure, the C4-MOP/PDA exhibited color transitions to external stimuli including temperature, organic solvent, and mechanical force. These results provide chiral polydiacetylene assemblies by using triterpenoid gelators and may open new developments in the design of smart responsive PDA materials.

\section{Conflicts of interest}

The authors declare that they have no conflict of interest.

\section{Acknowledgements}

This work is supported by the Open Fund of National Key Laboratory of Science and Technology on Advanced Composite (KZ42191814) and National Key R\&D Program of China (no. 2017YFD0200302).

\section{Notes and references}

1 S. Rondeau-Gagne, J. R. Neabo, M. Desroches, J. Larouche, J. Brisson and J.-F. Morin, J. Am. Chem. Soc., 2013, 135, 110-113.

2 Y.-Y. Xu, Z.-F. Ding, F.-Y. Liu, K. Sun, C. Dietlin, J. Lalevee and P. Xiao, ACS Appl. Mater. Interfaces, 2020, 12, 1658-1664.

3 K. Watanabe, H. Imai and Y. Oaki, Small, 2020, 16, 2004586.

4 D.-H. Park, W. Jeong, M. Seo, B. J. Park and J.-M. Kim, Adv. Funct. Mater., 2016, 26, 498-506.

5 G. Shin, M. I. Khazi and J.-M. Kim, Macromolecules, 2020, 53, 149-157.

6 G. Shin, M. I. Khazi and J.-M. Kim, Langmuir, 2020, 36, 13971-13980.

7 L. Polacchi, A. Brosseau, R. Metivier and C. Attain, Chem. Commun., 2019, 55, 14566-14569.

8 X. Qian and B. Stadler, Chem. Mater., 2019, 31, 1196-1222.

9 M. I. Khazi, W. Jeong and J.-M. Kim, Adv. Mater., 2018, 30, 1705310.

10 X. Chen, G. Zhou, X. Peng and J. Yoon, Chem. Soc. Rev., 2012, 41, 4610-4630. 
11 M. J. Seo, J. Song, C. Kantha, M. I. Khazi, U. Kundapur, J.-M. Heo and J.-M. Kim, Langmuir, 2018, 34, 8365-8373.

12 Y. Meng, J. Jiang and M. Liu, Nanoscale, 2017, 9, 7199-7206.

13 J. R. Neabo, K. I. S. Tohoundjona and J.-F. Morin, Org. Lett., 2011, 13, 1358-1361.

14 B. P. Krishnan, S. Mukherjee, P. M. Aneesh, M. A. G. Namboothiry and K. M. Sureshan, Angew. Chem., Int. Ed., 2016, 55, 2345-2349.

15 C. Chen, J. Chen, T. Wang and M. Liu, ACS Appl. Mater. Interfaces, 2016, 8, 30608-30615.

16 X. Huang, S. G. Jiang and M. Liu, J. Phys. Chem. B, 2005, 109, 114-119.

17 P. Duan, Y. Li and M. Liu, Sci. China: Chem., 2010, 53, 432-437.

18 Y. Gao, J. Hao, J. Wu, X. Zhang, J. Hu and Y. Ju, Nanoscale, 2015, 7, 13568-13575.

19 B. G. Bag, C. Garai, R. Majumdar and M. Laguerre, Struct. Chem., 2012, 23, 393-398.

20 J. Wang, M. Jin, C. Jin, C. Ye, Y. Zhou, R. Wang, H. Cui, W. Zhou and G. Li, Nat. Prod. Res., 2020, 34, 3313-3319.

21 Y. Gao, J. Hao, Q. Yan, F. Du, Y. Ju and J. Hu, ACS Appl. Mater. Interfaces, 2018, 10, 17352-17358.

22 B. G. Bag, C. Garai and S. Ghorai, RSC Adv., 2019, 9, 15190-15195.

23 B. G. Bag and S. S. Dash, Langmuir, 2015, 31, 13664-13672.

24 B. G. Bag, S. Das, S. N. Hasan and A. C. Barai, RSC Adv., 2017, 7, 18136-18143.

25 K. Vega-Granados, G. Belen Ramirez-Rodriguez, R. ContrerasMontoya, F. J. Ramirez, L. Palomo, A. Parra, J. M. DelgadoLopez, M. T. Lopez-Lopez and L. Alvarez de Cienfuegos, Mater. Chem. Front., 2019, 3, 2637-2646.

26 Y. Gao, J. Hao, J. Liu, Y. Liang, F. Du, J. Hu and Y. Ju, Mater. Chem. Front., 2019, 3, 308-313.
27 K. Sada, M. Takeuchi, N. Fujita, M. Numata and S. Shinkai, Chem. Soc. Rev., 2007, 36, 415-435.

28 H. Fan, H. Jiang, X. Zhu, M. Zhu, L. Zhang and M. Liu, Eur. Polym. J., 2019, 118, 146-152.

29 T. Hasegawa, S. Haraguchi, M. Numata, C. Li, A. Bae, T. Fujisawa, K. Kaneko, K. Sakuraic and S. Shinkai, Org. Biomol. Chem., 2005, 3, 4321-4328.

$30 \mathrm{~J} . \mathrm{Hu}, \mathrm{T}$. Zhu, C. He, Y. Zhang, Q. Zhang and G. Zou, J. Mater. Chem. C, 2017, 5, 5135-5142.

31 J. K. Lim, Y. Lee, K. Lee, M. Gong and S.-W. Joo, Chem. Lett., 2007, 36, 1226-1227.

32 M. Liu, L. Zhang and T. Wang, Chem. Rev., 2015, 115, 7304-7397.

33 E. Yashima, T. Matsushima and Y. Okamoto, J. Am. Chem. Soc., 1997, 119, 6345-6359.

34 B. Yue, L. Yin, W. Zhao, X. Jia, M. Zhu, B. Wu, S. Wu and L. Zhu, ACS Nano, 2019, 13, 12438-12444.

35 M. Okaniwa, Y. Oaki, S. Kaneko, K. Ishida, H. Maki and H. Imai, Chem. Mater., 2015, 27, 2627-2632.

36 N. Phonchai, C. Khanantong, F. Kielar, R. Traiphol and N. Traiphol, ACS Appl. Nano. Mater., 2019, 2, 4489-4498.

37 D. J. Ahn, E. Chae, G. S. Lee, H. Shim, T. Chang, K. Ahn and J. Kim, J. Am. Chem. Soc., 2003, 125, 8976-8977.

38 Y. Zhou, T. Yi, T. Li, Z. Zhou, F. Li, W. Huang and C. Huang, Chem. Mater., 2006, 18, 2974-2981.

39 Y. Gao, J. Lu, J. Wu, J. Hu and Y. Ju, $R S C A d v ., 2014,4$, 63539-63543.

40 Y.-J. Choi, S. Park, W.-J. Yoon, S.-I. Lim, J. Koo, D.-G. Kang, S. Park, N. Kim and K.-U. Jeong, Adv. Mater., 2020, 32, 2003980.

41 D. J. Ahn, S. Lee and J.-M. Kim, Adv. Funct. Mater., 2009, 19, 1483-1496. 